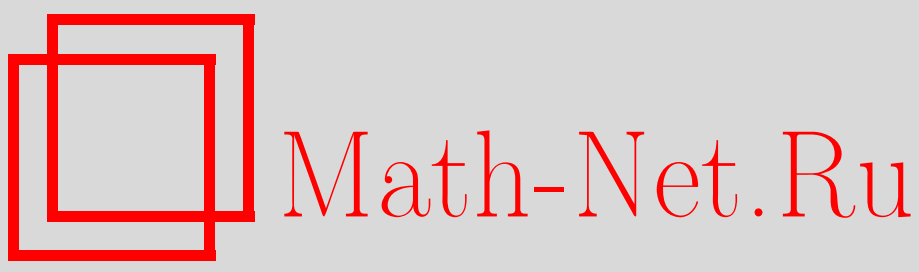

А. О. Смирнов, Об одном классе эллиптических потенциалов оператора Дирака, Матем. сб., 1997, том 188, номер 1, 109-128

DOI: https://doi.org/10.4213/sm190

Использование Общероссийского математического портала Math-Net.Ru подразумевает, что вы прочитали и согласны с пользовательским соглашением

http://www . mathnet.ru/rus/agreement

Параметры загрузки:

IP: 3.89 .197 .203

26 апреля 2023 г., $17: 12: 24$ 
УДК 517

\author{
А.О. Смирнов
}

\title{
Об одном классе эллиптических потенциалов оператора Дирака
}

В работе доказано, что существует класс конечнозонных потенциалов оператора Дирака и конечнозонных решений "расщепленного" нелинейного уравнения Шрёдингера, являющихся эллиптическими однозначньми мероморфнььми функциями по переменной $x$. Также доказано, что эволюция полюсов $x_{j}(t)$ этих эллиптических решений удовлетворяет динамике системы Калоджеро-Мозера.

Библиография: 38 названий.

\section{Введение}

Одно из развивающихся в настоящее время направлений теории конечнозонного интегрирования - теория редукции общих конечнозонных решений интегрируемых нелинейных уравнений (ИНУ) и/или конечнозонных потенциалов линейных дифференциальных операторов (ЛДО). Кратко стояшую задачу можно охарактеризовать следующим образом: необходимо найти условия, при которых общее конечнозонное решение ИНУ, выражающееся через многомерные тэта-функции римановых поверхностей больших родов, является эллиптической функцией. А поскольку любое ИНУ получается как условие коммутации двух Л ДО, то эллиптическое конечнозонное решение ИНУ в любой момент времени будет эллиптическим конечнозонньм потенциалом данных ЛДО. Причем в отличие от эллиптических конечнозонных потенциалов ЛДО, эллиптические конечнозонные решения ИНУ представляют интерес не только сами по себе, они также могут быть использованы для изучения динамики интегрируемых систем частиц (см., например, статьи [1]-[5], а также монографию [6]).

К настоящему времени разработано несколько методов построения эллиптических конечнозонных потенциалов ЛДО и конечнозонных решений ИНУ. Два метода основьваются на результатах работы [3]. В первом из них, реализованном для уравнений Кадомцева-Петвиашвили [3], Кортевега-де Фриза (КдФ) [7]-[10] и нелинейного уравнения Шрёдингера (НШІ) [11], используется специальный анзац для $\Psi$-функции - собственной функции вспомогательного линейного оператора (оператора Шрёдингера для уравнения КдФ и оператора Дирака для НШ). Алгебраические кривые, ассоциированные с эллиптическими конечнозонньми решениями, получаются как результат совместности некоторой переопределенной системы алгебраических уравнений от основного и дополнительных спектральных параметров и называются кривыми Кричевера.

Работа выполнена при финансовой поддержке Российского фонда фундаментальных исследований (грант № 95-01-00686). 
Этот же метод был применен к ряду ЛДО третьего порядка, не связанных ни с какими ИНУ [5], [12]-[14].

Метод, предложенный автором данной работы, основывается на выборе специальных анзацев для кривых Кричевера, не обращаясь непосредственно к линейной задаче. Он был успешно применен для нахождения эллиптических по $x$ и по $t$ peшений уравнения $\mathrm{K}$ д $[15]-[19]$, а также для описания новых довольно обширных классов эллиптических решений уравнений "sine-Gordon" [16], [19], [20], HШ, модифицированного уравнения КдФ и уравнения цепочки Тоды [19], [21], [22].

В самое последнее время появился еше один метод, примененный для исследования стационарных (без рассмотрения изоспектральной деформации в силу уравнения КдФ) эллиптических конечнозонных потенциалов оператора Шрёдингера [23]-[26].

При исследовании оператора Дирака (уравнение НШ) автором настоящей работы были использованы оба метода: первый - в статье [11], второй - в [21].

В работе [11] автором был получен анзац для кривых Кричевера $\Gamma=\{(k, \alpha)\}$, ассоциированных с эллиптическими многозонными потенциалами оператора Дирака

$$
-i \sigma_{3} \Psi_{x}+\mathfrak{U}(x, t) \Psi=\lambda \Psi,
$$

$\sigma_{3}$ - матрица Паули, $\mathfrak{U}(x, t)$ - потенциал,

$$
\begin{gathered}
\sigma_{3}=\left(\begin{array}{cc}
1 & 0 \\
0 & -1
\end{array}\right), \quad \mathfrak{U}(x, t)=\left(\begin{array}{cc}
0 & p(x, t) \\
q(x, t) & 0
\end{array}\right), \\
\mathfrak{U}(x, t)=\sum_{j=1}^{n} \mathfrak{U}_{j}^{0}\left(x-x_{j}(t), t\right), \\
p_{j}^{0}(x, t)=-\frac{i}{\mu_{j}(t)} \frac{\sigma\left(x+2 \alpha_{0}\right)}{\sigma(x) \sigma\left(2 \alpha_{0}\right)} \exp \left\{-\zeta\left(2 \alpha_{0}\right) x\right\}, \\
q_{j}^{0}(x, t)=-i \mu_{j}(t) \frac{\sigma\left(x-2 \alpha_{0}\right)}{\sigma(x) \sigma\left(2 \alpha_{0}\right)} \exp \left\{\zeta\left(2 \alpha_{0}\right) x\right\},
\end{gathered}
$$

$\sigma(x), \zeta(x)$ - эллиптические функции Вейерштрасса [27].

При этом $\Psi$-функция есть линейная комбинация однозонных $\Psi$-функций оператора Дирака с коэффициентами, зависящими от дополнительных спектральных параметров $k$ и $\alpha$ [11],

$$
\Psi=\sum_{j=1}^{n} a_{j}(k, \alpha, t) \Psi_{j}^{0}\left(x-x_{j}(t), \alpha, t\right) e^{k x / 2},
$$

а функции $p(x, t), q(x, t)$ - эллиптические функции второго рода по переменной $x$ (см., например, [27]).

В то же время в статье [21] с помощью второго метода было найдено два различных анзаца кривых Кричевера, ассоциированњых с эллиптическими по $x$ решениями уравнения НШ. И каждое такое решение при любом $t$ будет эллиптическим конечнозонным потенциалом оператора Дирака.

Один единственный анзац кривых Кричевера, полученный первым методом в работе [11], соответствует анзацу II статьи [21]. Следовательно, возможен один из 
двух вариантов. Или анзац I кривых Кричевера [21], ассоциированных с эллиптическими по $x$ решениями уравнения НШ, пуст, или сушествует еше один, новый, не сводящийся к (0.2), (0.3) класс эллиптических потенциалов оператора Дирака.

В данной работе мы покажем, что анзац I не пуст, и опишем новый класс эллиптических потенциалов оператора Дирака, являющихся, в отличие от потенциалов анзаца II, не эллиптическими функциями второго рода, а эллиптическими мероморфными (однозначными) функциями. Эволюция полюсов $x_{j}(t)$ этих эллиптических потенциалов будет в силу уравнения НШ описывать динамику интегрируемой системы частиц Калоджеро-Мозера [1]-[6] $\left(\dot{x} \equiv \partial_{t} x\right)$ :

$$
\ddot{x}_{j}=4 \sum_{k \neq j} \wp^{\prime}\left(x_{j}-x_{k}\right),
$$

$\wp(x)$ - эллиптическая функция Вейерштрасса [27].

Динамика полюсов конечнозонного эллиптического решения уравнения НШ (анзац II) будет рассмотрена отдельно.

Автор благодарит А.Р. Итса и М. А. Салля за полезные обсуждения.

\section{1. Конечнозонные решения нелинейного уравнения Шрёдингера и модифицированного уравнения Кортевега-де Фриза}

Обшие конечнозонные решения "расшепленного"

$$
\left\{\begin{array}{l}
i p_{t}+p_{x x}-2 p^{2} q=0 \\
i q_{t}-q_{x x}+2 p q^{2}=0
\end{array}\right.
$$

уравнения НШ, давно и хорошо известны [28]-[31], [10], [19], [21], [22]. Они строятся по гиперэллиптической поверхности (ГП) рода $g$ :

$$
\Gamma: w^{2}=\prod_{j=1}^{2 g+2}\left(\lambda-\lambda_{j}\right)=\lambda^{2 g+2}+\sum_{j=1}^{2 g+2} \gamma_{j} \lambda^{2 g+2-j},
$$

точки ветвления которой $\left(\lambda=\lambda_{j}, j=1, \ldots, 2 g+2\right)$ являются краями зон спектра оператора Дирака (0.1). Бесконечно удаленной точке спектра соответствуют две различные точки $\mathscr{P}_{\infty}^{ \pm}$данной ГП.

Выберем на Г базис циклов $\gamma^{t}=\left(a_{1}, \ldots, a_{g}, b_{1}, \ldots, b_{g}\right)$ с матрицей пересечения $C_{0}=\left(\begin{array}{rr}0 & \mathrm{I} \\ -\mathrm{I} & 0\end{array}\right)$. Ему соответствует нормированньй базис голоморфных дифференциалов $d \mathscr{U}_{j}$,

$$
\oint_{a_{k}} d \mathscr{U}_{j}=\delta_{k j}, \quad k, j=1, \ldots, g .
$$

Хорошо известно, что матрица периодов

$$
B_{j k}=\oint_{b_{j}} d \mathscr{U}_{k}, \quad j, k=1, \ldots, g
$$

есть симметрическая матрица с положительно определенной мнимой частью. 
По матрице периодов $B$ построим тэта-функцию с характеристиками $\alpha, \beta \in \mathbb{R}^{g}$ :

$$
\begin{gathered}
\Theta[\alpha ; \beta](z \mid B) \equiv \Theta[\alpha ; \beta](z)=\sum_{\substack{m \in \mathbb{Z}^{g} \\
\Theta[0 ; 0](z \mid B) \equiv \Theta(z \mid B) \equiv \Theta(z),}} \exp \{\pi i\langle B(m+\alpha), m+\alpha\rangle+2 \pi i\langle m+\alpha, z+\beta\rangle\},
\end{gathered}
$$

где $z \in \mathbb{C}^{g}$, суммирование ведется по целочисленной $g$-мерной решетке, а угловые скобки $\langle$,$\rangle обозначают евклидово скалярное произведение.$

Введем на $\Gamma$ нормированные абелевы интегралы второго $-\Omega_{1}(\mathscr{P}), \Omega_{2}(\mathscr{P})$, $\Omega_{3}(\mathscr{P})$, и третьего - $\omega(\mathscr{P})$, родов, $\mathscr{P} \in \Gamma, \mathscr{P}=(w, \lambda)$, с асимптотикой в бесконечно удаленных точках $\mathscr{P} \underset{\infty}{ \pm}$ :

$$
\begin{gathered}
\oint_{a_{k}} d \Omega_{1}=\oint_{a_{k}} d \Omega_{2}=\oint_{a_{k}} d \Omega_{3}=\oint_{a_{k}} d \omega=0, \quad k=1, \ldots, g, \\
\Omega_{1}(\mathscr{P})= \pm i\left(\lambda-K_{1}+R_{1} \lambda^{-1}+O\left(\lambda^{-2}\right)\right), \quad \mathscr{P} \rightarrow \mathscr{P}_{\infty}^{ \pm}, \\
\Omega_{2}(\mathscr{P})=\mp i\left(2 \lambda^{2}+K_{2}+O\left(\lambda^{-1}\right)\right), \quad \mathscr{P} \rightarrow \mathscr{P}_{\infty}^{ \pm}, \\
\Omega_{3}(\mathscr{P})= \pm i\left(4 \lambda^{3}-K_{3}+O\left(\lambda^{-1}\right)\right), \quad \mathscr{P} \rightarrow \mathscr{P}_{\infty}^{ \pm}, \\
\omega(\mathscr{P})=\mp\left(\ln \lambda-\ln K_{0}+O\left(\lambda^{-1}\right)\right), \quad \mathscr{P} \rightarrow \mathscr{P}_{\infty}^{ \pm}, \\
w= \pm\left(\lambda^{g+1}+O\left(\lambda^{g}\right)\right), \quad \mathscr{P} \rightarrow \mathscr{P}_{\infty}^{ \pm} .
\end{gathered}
$$

Если мы теперь обозначим через $2 \pi i U, 2 \pi i V, 2 \pi i W$ векторы $b$-периодов абелевых интегралов второго рода $\Omega_{1}(\mathscr{P}), \Omega_{2}(\mathscr{P})$ и $\Omega_{3}(\mathscr{P})$ соответственно, то функция

$$
\Psi(\mathscr{P}, x, t, y)=\left(\begin{array}{ll}
\psi_{1}(\mathscr{P}, x, t, y) & \psi_{1}(\tau \mathscr{P}, x, t, y) \\
\psi_{2}(\mathscr{P}, x, t, y) & \psi_{2}(\tau \mathscr{P}, x, t, y)
\end{array}\right)
$$

где $\tau$ - гиперэллиптическая инволюция $\tau:(w, \lambda) \rightarrow(-w, \lambda)$,

$$
\begin{aligned}
\psi_{1}(\mathscr{P}, x, t, y)= & \frac{\Theta(\mathscr{U}(\mathscr{P})+U x+V t+W y-X) \Theta(Z)}{\Theta(\mathscr{U}(\mathscr{P})-X) \Theta(U x+V t+W y+Z)} \\
& \times \exp \left\{\Omega_{1}(\mathscr{P}) x+\Omega_{2}(\mathscr{P}) t+\Omega_{3}(\mathscr{P}) y+i \Phi(x, t, y)\right\} \\
\psi_{2}(\mathscr{P}, x, t, y)= & \mu \frac{\Theta(\mathscr{U}(\mathscr{P})+U x+V t+W y+D-X) \Theta(Z-D)}{\Theta(\mathscr{U}(\mathscr{P})-X) \Theta(U x+V t+W y+Z)} \\
& \times \exp \left\{\Omega_{1}(\mathscr{P}) x+\Omega_{2}(\mathscr{P}) t+\Omega_{3}(\mathscr{P}) y-i \Phi(x, t, y)+\omega(\mathscr{P})\right\}
\end{aligned}
$$

при любом $\mu \neq 0$ и при любых $t, y$ будет являться собственной функцией оператора Дирака (0.1) с потенциалом

$$
\begin{aligned}
& p(x, t, y)=\frac{2 K_{0}}{\mu} \frac{\Theta(Z) \Theta(U x+V t+W y+Z-D)}{\Theta(Z-D) \Theta(U x+V t+W y+Z)} \exp \{2 i \Phi(x, t, y)\} \\
& q(x, t, y)=2 \mu K_{0} \frac{\Theta(Z-D) \Theta(U x+V t+W y+Z+D)}{\Theta(Z) \Theta(U x+V t+W y+Z)} \exp \{-2 i \Phi(x, t, y)\}
\end{aligned}
$$

по переменной $t$ удовлетворяющим уравнению НШ (1.1), а по переменной $y$ - "расщепленному" модифицированному уравнению Кортевега-де Фриза (МКд $\Phi)$

$$
\left\{\begin{array}{l}
p_{y}+p_{x x x}-6 p q p_{x}=0 \\
q_{y}+q_{x x x}-6 p q q_{x}=0 .
\end{array}\right.
$$


Здесь $D$ - вектор абелевых голоморфных интегралов, вычисленных вдоль пути, соединяюшего точки $\mathscr{P}_{\infty}^{-}$и $\mathscr{P}_{\infty}^{+}$и не пересекаюшего ни один из базисных циклов,

$$
\begin{gathered}
D=\mathscr{U}\left(\mathscr{P}_{\infty}^{+}\right)-\mathscr{U}\left(\mathscr{P}_{\infty}^{-}\right), \quad \Phi(x, t, y)=K_{1} x+K_{2} t+K_{3} y \\
X=\mathscr{K}+\sum_{j=1}^{g} \mathscr{U}\left(\mathscr{P}^{j}\right), \quad Z=\mathscr{U}\left(\mathscr{P}_{\infty}^{+}\right)-X
\end{gathered}
$$

$\mathscr{K}$ - вектор римановых констант [31]-[33]; $\mathscr{P}^{j}, j=1, \ldots, g,-$ неспециальный дивизор. При этом будет справедливым соотношение [28]-[31], [10]

$$
u(x, t, y) \equiv p(x, t, y) q(x, t, y)=-\partial_{x}^{2} \ln \Theta(U x+V t+W y+Z)-2 R_{1},
$$

которое обобщает на случай $g>1$ известную формулу связи между эллиптическими функциями Якоби и Вейерштрасса.

\section{2. Редукция тэта-функций}

Пусть риманова поверхность Г является $n$-листным накрытием над эллиптической поверхностью $\Gamma_{0}\left(\mathfrak{s}: \Gamma \rightarrow \Gamma_{0}\right)$,

$$
\Gamma_{0}:\left\{\wp^{\prime}(\alpha)\right\}^{2}=4 \wp^{3}(\alpha)-g_{2} \wp(\alpha)-g_{3} \equiv 4 \prod_{j=1}^{3}\left(\wp(\alpha)-e_{j}\right) .
$$

Отображение $\mathfrak{s}$ порождает отображения гомологий и якобианов

$$
\widehat{\mathfrak{s}} \gamma=\left(\begin{array}{cc}
S & P \\
Q & R
\end{array}\right) \gamma_{0}, \quad \mathfrak{s}^{*} d \mathscr{U}^{0}=\langle T, d \mathscr{U}\rangle,
$$

удовлетворяюшие следуюшим условиям [7], [16]

$$
\begin{gathered}
T=S+B_{0} P, \quad\langle S, R\rangle-\langle Q, P\rangle=n, \\
B S-Q=B_{0}(R-B P),
\end{gathered}
$$

где $B, B_{0}$ - матрицы периодов поверхностей $\Gamma, \Gamma_{0}$.

Теорема 1 [16]. Пусть существуют на Г и Г второго рода $d \Omega$ и $d \Omega^{0}$, нормированнье в соответствующих базисах циклов, с векторами b-периодов $2 \pi i U$ и $2 \pi i U^{0}$ и связанные соотношением

$$
\mathfrak{s}^{*} d \Omega^{0} \equiv d \Omega+2 \pi i U^{0}\langle P, d \mathscr{U}\rangle
$$

с точностью до дифференциала от мероморфной функции.

Тогда верны следующие равенства:

$$
\begin{aligned}
& \Theta(U z+\Delta \mid B)=\chi \exp \left\{a\left(U^{0} z\right)^{2}+b U^{0} z\right\} \prod_{j=1}^{n} \Theta\left(U^{0} z+Z_{j} \mid B_{0}\right), \\
& \Theta(U z+\Delta \mid B)=\exp \left\{a\left(U^{0} z\right)^{2}+b U^{0} z\right\} \sum_{j=0}^{n-1} \chi_{j} \Theta[j / n ; 0]\left(n U^{0} z+\delta \mid n B_{0}\right),
\end{aligned}
$$


әде п - число листов накрытия,

$$
\begin{gathered}
a=\pi i\langle P, R-B P\rangle, \quad b=\pi i\langle P, 2 \Delta-R\rangle, \quad \sum_{j=1}^{n} Z_{j}-\delta \in \mathbb{Z}, \\
\delta=\left\langle S+B_{0} P, \Delta\right\rangle+\frac{1}{2}\left\{\langle S, Q\rangle-B_{0}\langle P, R\rangle\right\},
\end{gathered}
$$

$\chi, \chi_{j}$ - некоторые постоянные.

ДокАЗАТЕЛЬСтво. Из равенства

$$
\int_{\mathscr{L}} \mathfrak{s}^{*} d \Omega^{0}=\int_{\widehat{\mathfrak{s}} \mathscr{L}} d \Omega^{0}
$$

где $\mathscr{L}$ есть произвольный путь на $Г$, вытекает, что векторы $b$-периодов этих дифференциалов удовлетворяют уравнению

$$
U=U^{0}(R-B P)
$$

Редукция (2.5)-(2.7) может быть доказана с помошью закона преобразования тэта-функции при с двиге на вектор решетки [31], [32], [34], [35], [10], [16], определения и свойств одномерной тэта-функции $n$-го порядка [32], [34], [35], [16] и соотношений (2.2), (2.3), (2.8).

ЗАмЕчАниЕ 1 . Ниже мы будем использовать следующие равенства, фиксирующие базис циклов и абелевы дифференциалы на $\Gamma_{0}$ (применяются стандартные обозначения теории эллиптических функций [27]):

$$
\begin{gathered}
\int_{a^{0}} d \alpha=2 \omega, \quad \int_{b^{0}} d \alpha=2 \omega^{\prime}, \quad \operatorname{Im} \frac{\omega^{\prime}}{\omega}>0 \\
d \mathscr{U}^{0}=\frac{1}{2 \omega} d \alpha, \quad B_{0}=\frac{\omega^{\prime}}{\omega}, \quad \Omega_{1}^{0}(\alpha)=\zeta(\alpha)-\frac{\eta}{\omega} \alpha, \quad U_{1}^{0}=-\frac{1}{2 \omega}, \\
\Omega_{2}^{0}(\alpha)=\frac{1}{2} \zeta\left(\alpha-\alpha_{0}\right)+\frac{1}{2} \zeta\left(\alpha+\alpha_{0}\right)-\frac{\eta}{\omega} \alpha, \quad U_{2}^{0}=-\frac{1}{2 \omega} .
\end{gathered}
$$

В этом случае

$$
\begin{aligned}
\frac{\Theta\left(U^{0} z+Z_{j} \mid B_{0}\right)}{\Theta\left(0 \mid B_{0}\right)}= & -\frac{\sigma\left(z-2 \omega Z_{j}-\omega-\omega^{\prime}\right)}{\sigma\left(\omega+\omega^{\prime}\right)} \\
& \times \exp \left\{-\frac{\eta}{2 \omega}\left(z-2 \omega Z_{j}\right)^{2}+\left(\eta+\eta^{\prime}\right)\left(z-2 \omega Z_{j}\right)\right\}
\end{aligned}
$$

СЛЕДСТВИЕ 1. В условиях теоремы 1

$$
\begin{aligned}
\left\langle S+B_{0} P, \mathscr{U}\left(\mathscr{P}_{2}\right)-\mathscr{U}\left(\mathscr{P}_{1}\right)\right\rangle & =\int_{\mathscr{P}_{1}}^{\mathscr{P}_{2}} \mathfrak{s}^{*} d \mathscr{U}^{0} \\
& =\int_{\mathfrak{s}\left(\mathscr{P}_{1}\right)}^{\mathfrak{s}\left(\mathscr{P}_{2}\right)} d \mathscr{U}^{0}=\frac{1}{2 \omega}\left(\alpha_{2}-\alpha_{1}\right)+M_{1}+M_{2} B_{0},
\end{aligned}
$$

где $M_{1}, M_{2} \in \mathbb{Z}$ - челье постоянные, зависящие от пути интегрирования; $\alpha_{1}=\mathfrak{s}\left(\mathscr{P}_{1}\right), \alpha_{2}=\mathfrak{s}\left(\mathscr{P}_{2}\right)$. 
СлЕДСТвИЕ 2. В условиях теоремы 1 функиия

$$
H(z)=\frac{\Theta\left(U z+\Delta_{1} \mid B\right)}{\Theta\left(U z+\Delta_{2} \mid B\right)}
$$

является әллиптической функцией второго рода, имеющей по $n$ нулей и полюсов на торе $\Gamma_{0}$.

ДокАЗАТЕЛЬСТво легко следует из законов преобразования функции $H(z)$ при сдвиге на периоды тора:

$$
\begin{aligned}
H(z+2 \omega) & =\exp \left\{-2 \pi i\left\langle\Delta_{1}-\Delta_{2}, P\right\rangle\right\} H(z), \\
H\left(z+2 \omega^{\prime}\right) & =\exp \left\{2 \pi i\left\langle\Delta_{1}-\Delta_{2}, S\right\rangle\right\} H(z)
\end{aligned}
$$

и соотношений $(2.5),(2.7),(2.10)$.

СлЕДСТвИЕ 3. В условиях теоремь 1 функция

$$
H_{1}(z)=-\partial_{z}^{2} \ln \Theta(U z+\Delta \mid B)
$$

является әллиптической функцией, имеющей на торе $\Gamma_{0} n$ полюсов второго порядка $\left(z_{j}=2 \omega Z_{j}+\omega+\omega^{\prime}\right)$,

$$
H_{1}(z)=\sum_{j=1}^{n} \wp\left(z-z_{j}\right)+\frac{n \eta}{\omega}-\frac{a}{2 \omega^{2}} .
$$

ДОКАЗАТЕЛЬСТво следует из соотношений $(2.5),(2.7),(2.10)$ и свойств эллиптических функций.

\section{3. Кривые Кричевера для эллиптических решений уравнений НШ и МКдФ}

Анзац I. Рассмотрим риманову поверхность $\Gamma=\{(k, \alpha)\}$ рода $g, n$-листно, $n \geqslant 3$, накрывающую эллиптическую $\Gamma_{0}$ и удовлетворяющую следующим требованиям [21].

1)

$$
\Gamma: \mathscr{R}(k, \alpha) \equiv k^{n}+\sum_{j=2}^{n} r_{j}(\alpha) k^{n-j}=0
$$

где $r_{j}(\alpha)$ - эллиптические функции от $\alpha \in \Gamma_{0}$ с полюсами в точке $\alpha=0$.

$2)$ В окрестности $\alpha=0$ функция $\mathscr{R}(k, \alpha)$ представима в виде

$$
\mathscr{R}(k, \alpha)=\left(k-\frac{n-2}{2 \alpha}+b_{n}(\alpha)\right)\left(k-\frac{n-2}{2 \alpha}-b_{n}(-\alpha)\right) \prod_{j=1}^{n-2}\left(k+\frac{1}{\alpha}+b_{j}(\alpha)\right)
$$

$b_{j}(\alpha)$ - регулярные функции $\alpha$.

3) На Г существует инволюция

$$
\tau:(k, \alpha) \rightarrow(-k,-\alpha),
$$

имеюшая $2 g+2$ неподвижные точки. 
ЗАмечаниЕ 2. Из пунктов 3) и 2) следует, что поверхность Г является гиперэллиптической, и что пара выделенных точек $\mathscr{P}_{\infty}^{ \pm}$переходят друг в друга при гиперэллиптической инволюции $\tau$.

Замечание 3. Используя формулу Римана-Гурвища [32], [7], [10], нетрудно получить, что род $g$ поверхности Г (2.1), (3.1)-(3.3) равен $p+1$, где $2 p$ - число нулей функции $\partial_{k} \mathscr{R}(k, \alpha)$, не лежащих "над" $\alpha=0$ и отличных от нулей функции $\partial_{\alpha} \mathscr{R}(k, \alpha)$ (с учетом кратности).

Tеорема 2 [21]. Решения $p(x, t, y)$ и $q(x, t, y)$ уравнений НШІ (1.1) и МКдФ (1.6), построенные по ГП $\Gamma_{g}^{n}(2.1),(3.1)-(3.3)$ и отмеченным точкам $\mathscr{P} \pm$ “над" $\alpha=0$, могут быть выражсни с точностью до әкспоненциальных множителей через әллиптические б-функиии Вейеритрасса ( $с$ периодами накрываемой поверхности (2.1)) от переменной $x$. При этом функиия $u(x, t, y)$ (1.7) будет әллиптической функиией по $x$ :

$$
u(x, t, y)=\sum_{j=1}^{n} \wp\left(x-x_{j}(t, y)\right)-\varkappa
$$

Здесь $\wp(x)$ - эллиптическая функция Вейеритрасса с периодами накрываемой поверхности (2.1), $x_{j}(t, y)$ - нули тәта-функции $\Theta(U x+V t+W y+Z \mid B)$ как функиии от переменной $x, \varkappa$ - некоторая постоянная.

ДокАЗАТЕльство. Выберем в качестве спектральной переменной функцию $\lambda(\mathscr{P}), \mathscr{P} \in \Gamma$, инвариантную относительно инволюции $\tau(3.3)$ и имеюшую пару простых полюсов в точках $\mathscr{P}_{\infty}^{ \pm}$вида

$$
\lambda=\mp \frac{i n}{2 \alpha}+O(1), \quad \mathscr{P} \rightarrow \mathscr{P}_{\infty}^{ \pm}
$$

Из (3.5) и (1.3) следует, что нормированный абелев дифференциал второго рода $d \Omega_{1}$ будет иметь следуюшую асимптотику в окрестности точек $\mathscr{P}_{\infty}^{ \pm}$

$$
d \Omega_{1}= \pm i\left(1+O\left(\lambda^{-2}\right)\right) d \lambda=\left(-\frac{n}{2 \alpha^{2}}+O(1)\right) d \alpha
$$

Применяя теорему Лиувилля, нетрудно показать, что нормированные абелевы дифференциалы второго рода $d \Omega_{1}(1.3)$ и $d \Omega_{1}^{0}(2.9)$, определенные на $\Gamma$ и $\Gamma^{0}$, соответственно, связаны соотношением

$$
\mathfrak{s}^{*} d \Omega_{1}^{0} \equiv d \Omega_{1}-d k
$$

с точностью до голоморфных дифференциалов.

Теперь легко видеть, что утверждение теоремы 2 является следствием теоремы 1 , равенства (3.7) и формул связи эллиптических функций Вейерштрасса с одномерными тэта-функциями. 
ЗАмечАниЕ 4. Также к этому классу поверхностей мы будем относить двулистные накрытия, удовлетворяюшие условиям 1), 3) и не имеюшие "над" $\alpha=0$ неподвижных точек. В этом случае связь абелевых дифференциалов $d \Omega_{1}(1.3)$ и $d \Omega_{1}^{0}(2.9)$ описывается равенством

$$
\mathfrak{s}^{*} d \Omega_{1}^{0}=d \Omega_{1}+d \text { (holom.). }
$$

Анзац II. Рассмотрим риманову поверхность $\Gamma=\{(k, \alpha)\}$ рода $g, n$-листно, $n \geqslant 2$, накрываюшую тор $\Gamma_{0}(2.1)$ и удовлетворяюшую следуюшим требованиям [21].

1)

$$
\Gamma: \mathscr{R}(k, \alpha) \equiv k^{n}+\sum_{j=2}^{n} r_{j}(\alpha) k^{n-j}=0,
$$

где $r_{j}(\alpha)$ - эллиптические функции от $\alpha \in \Gamma_{0}$ с полюсами в точках $\alpha= \pm \alpha_{0}$. 2) В окрестности $\alpha= \pm \alpha_{0}$ функция $\mathscr{R}(k, \alpha)$ представима в виде

$$
\begin{gathered}
\mathscr{R}(k, \alpha)=\left(k-\frac{n-1}{\alpha \mp \alpha_{0}}+b_{n}^{ \pm}\left(\alpha \mp \alpha_{0}\right)\right) \prod_{j=1}^{n-1}\left(k+\frac{1}{\alpha \mp \alpha_{0}}+b_{j}^{ \pm}\left(\alpha \mp \alpha_{0}\right)\right), \\
b_{j}^{ \pm}\left(\alpha \mp \alpha_{0}\right) \text { - регулярные функции от } \alpha \mp \alpha_{0} .
\end{gathered}
$$

3) На Г существует инволюция

$$
\tau:(k, \alpha) \rightarrow(-k,-\alpha)
$$

имеюшая $2 g+2$ неподвижные точки.

ЗАмечаниЕ 5 . Из пунктов 3) и 2) следует, что поверхность Г является гиперэллиптической, и что выделенные точки $\mathscr{P}_{\infty}^{ \pm}$, расположенные "над" $\alpha= \pm \alpha_{0}$, переходят друг в друга при гиперэллиптической инволюции $\tau$.

ЗАмечание 6. Используя формулу Римана-Гурвица [32], [7], [10], нетрудно получить, что род $g$ поверхности $\Gamma(2.1),(3.8)-(3.10)$ равен $p+1$, где $2 p$-число нулей функции $\partial_{k} \mathscr{R}(k, \alpha)$, не лежаших "над" $\alpha= \pm \alpha_{0}$ и отличных от нулей функции $\partial_{\alpha} \mathscr{R}(k, \alpha)$ (с учетом кратности).

Tеорема 3 [21]. Решения $p(x, t, y)$ и $q(x, t, y)$ уравнений НШ (1.1) и МКдФ (1.6), построенные по ГП $\Gamma_{g}^{n}(2.1),(3.8)-(3.10)$ и отмеченным точкам $\mathscr{P}_{\infty}^{ \pm}$ "над" $\alpha= \pm \alpha_{0}$, могут быть выражень, с точностью до әкспоненциальных множителей, через әллиптические б-функиии Вейерштрасса ( одами накрываемой поверхности (2.1)) от переменной $x$. При этом функиия $u(x, t, y)(1.7)$ будет әллиптической функцией по $x$ (см. (3.4)).

ДокАЗАТЕльство. Выберем в качестве спектральной переменной функцию $\lambda(\mathscr{P}), \mathscr{P} \in \Gamma$, инвариантную относительно инволюции $\tau(3.3)$ и имеющую пару простых полюсов в точках $\mathscr{P}_{\infty}^{ \pm}$вида

$$
\lambda=\mp \frac{i n}{2\left(\alpha \mp \alpha_{0}\right)}+O(1), \quad \mathscr{P} \rightarrow \mathscr{P}_{\infty}^{ \pm} .
$$


Из (3.11) и (1.3) следует, что нормированный абелев дифференциал второго рода $d \Omega_{1}$ будет иметь следуюшую асимптотику в окрестности точек $\mathscr{P}_{\infty}^{ \pm}$

$$
d \Omega_{1}= \pm i\left(1+O\left(\lambda^{-2}\right)\right) d \lambda=\left(-\frac{n}{2\left(\alpha \mp \alpha_{0}\right)^{2}}+O(1)\right) d \alpha .
$$

Теперь легко видеть, что нормированные абелевы дифференциалы второго рода $d \Omega_{1}(1.3)$ и $d \Omega_{2}^{0}(2.9)$, определенные на $\Gamma$ и $\Gamma^{0}$ соответственно, связаны соотношением

$$
\mathfrak{s}^{*} d \Omega^{0} \equiv d \Omega_{1}-\frac{1}{2} d k,
$$

которое и доказывает утверждение теоремы 3.

\section{4. Кривая Кричевера. Анзац I. Пример 1}

Спектральная кривая $\Gamma_{2}^{5}=\{(k, \alpha)\}$, ассоциированная с одним из эллиптических двухзонных решений уравнения НШ (анзац I), задается уравнением:

$$
\begin{aligned}
\mathscr{R}(k, \alpha)= & k^{5}-\frac{15}{4}\left\{\wp(\alpha)+3 a^{2}\right\} k^{3}+\frac{5}{8} \wp^{\prime}(\alpha) k^{2} \\
& +\frac{15}{4}\left\{\wp^{2}(\alpha)+9 a^{2} \wp(\alpha)-\frac{g_{2}}{30}\right\} k-\frac{9}{8} \wp^{\prime}(\alpha)\left\{\wp(\alpha)+10 a^{2}\right\}=0 .
\end{aligned}
$$

Здесь

$$
\begin{aligned}
& g_{2}=\frac{300 a^{4}}{s^{3}}\left(2-s^{3}\right)\left(2 s^{3}-1\right), \\
& g_{3}=\frac{500 a^{6}}{s^{6}}\left(1-14 s^{3}+24 s^{6}-14 s^{9}+s^{12}\right),
\end{aligned}
$$

$a, s$-вспомогательные параметры решения.

Каноническая форма гиперэллиптической кривой $\Gamma_{2}^{5}$ имеет вид:

$$
w^{2}=\left(\lambda^{3}+A_{1} \lambda^{2}+C_{1}\right)\left(\lambda^{3}+A_{2} \lambda^{2}+B_{2} \lambda+C_{2}\right),
$$

где

$$
\begin{gathered}
A_{1}=6 a, \quad C_{1}=\frac{4 a^{3}}{s^{3}}\left(25-58 s^{3}+25 s^{6}\right), \\
A_{2}=12 a, \quad B_{2}=-27 a^{2}, \quad C_{2}=\frac{a^{3}}{s^{3}}\left(125-236 s^{3}+125 s^{6}\right) .
\end{gathered}
$$

Связь между различными параметризациями кривой $\Gamma_{2}^{5}$ осушествляется с помошью соотношений

$$
\begin{aligned}
\wp^{\prime}(\alpha) & =\frac{16 i w\left(\lambda^{2}+A_{4} \lambda+B_{4}\right)\left(\lambda^{4}+A_{5} \lambda^{3}+B_{5} \lambda^{2}+C_{5} \lambda+D_{5}\right)}{125\left(\lambda^{3}+A_{1} \lambda^{2}+C_{1}\right)^{2}}, \\
\wp(\alpha) & =-\frac{4\left(\lambda^{5}+A_{3} \lambda^{4}+B_{3} \lambda^{3}+C_{3} \lambda^{2}+D_{3}\right)}{25\left(\lambda^{3}+A_{1} \lambda^{2}+C_{1}\right)}, \\
k & =\frac{3 i w(\lambda+4 a)}{5\left(\lambda^{3}+A_{1} \lambda^{2}+C_{1}\right)}, \quad \lambda=-\frac{\partial_{k} \mathscr{R}(k, \alpha)}{15 a\left(k^{2}-\wp(\alpha)+c\right)} .
\end{aligned}
$$


Здесь

$$
\begin{gathered}
A_{3}=15 a, \quad B_{3}=\frac{145}{4} a^{2}, \quad C_{3}=\frac{5 a^{3}}{4 s^{3}}\left(125-272 s^{3}+125 s^{6}\right), \\
D_{3}=\frac{6 a^{5}}{s^{3}}\left(625-1125 s^{3}+625 s^{6}\right), \quad c=\frac{5 a^{2}}{4 s^{3}}\left(1-10 s^{3}+s^{6}\right), \\
A_{4}=-\frac{a}{2 s}\left(5-11 s+5 s^{2}\right), \quad B_{4}=\frac{a^{2}}{s^{2}}\left(5-11 s+5 s^{2}\right)\left(5+4 s+5 s^{2}\right), \\
A_{5}=\frac{a}{2 s}\left(5+22 s+5 s^{2}\right), \quad B_{5}=-\frac{3 a^{2}}{4 s^{2}}\left(25-65 s-48 s^{2}-65 s^{3}+25 s^{4}\right), \\
C_{5}=\frac{a^{3}}{2 s^{3}}\left(5-8 s+5 s^{2}\right)\left(25+55 s+96 s^{2}+55 s^{3}+25 s^{4}\right), \\
D_{5}=\frac{a^{4}}{s^{4}}\left(25-20 s-9 s^{2}-20 s^{3}+25 s^{4}\right)\left(25+55 s+96 s^{2}+55 s^{3}+25 s^{4}\right) .
\end{gathered}
$$

Накрытие (4.1) связано с редукцией к эллиптическим дифференциалам абелева голоморфного дифференциала

$$
\frac{5 i \lambda d \lambda}{2 w}=d \alpha
$$

и абелева дифференциала второго рода с простыми полюсами в точках $\mathscr{P}_{\infty}^{ \pm}$

$$
d \Omega_{1}=i\left\{\lambda^{3}+A_{6} \lambda^{2}+B_{6} \lambda+C_{6}\right\} \frac{d \lambda}{w}=-\wp(\alpha) d \alpha+d k,
$$

где

$$
A_{6}=9 a, \quad B_{6}=\frac{3}{2} a^{2}, \quad C_{6}=\frac{3 a^{3}}{s^{3}}\left(25-58 s^{3}+25 s^{6}\right) .
$$

\section{5. Кривая Кричевера. Анзац I. Пример 2}

Спектральная кривая $\widetilde{\Gamma}_{2}^{5}$, ассоциированная со вторым эллиптическим двухзоннгм решением уравнения НШ, задается уравнением:

$$
\begin{aligned}
\widetilde{\mathscr{R}}(k, \alpha)= & k^{5}-\frac{15}{4}\{\wp(\alpha)+H\} k^{3}+\frac{5}{8} \wp^{\prime}(\alpha) k^{2} \\
& +\frac{15}{4}\left\{\wp(\alpha)-e_{j}\right\}\left\{\wp(\alpha)+H+\frac{2}{5} e_{j}\right\} k-\frac{9}{8} \wp^{\prime}(\alpha)\left\{\wp(\alpha)-e_{j}\right\}=0 .
\end{aligned}
$$

Здесь

$$
\begin{aligned}
& H=-a^{2} \frac{125+430 t^{2}-27 t^{4}}{\left(t^{2}-5\right)^{2}}, \\
& e_{k}=5 a^{2} \frac{125-110 t^{2}-72 t^{3}+9 t^{4}}{\left(t^{2}-5\right)^{2}}, \\
& e_{j}=-10 a^{2} \frac{125-110 t^{2}+9 t^{4}}{\left(t^{2}-5\right)^{2}}, \\
& e_{l}=5 a^{2} \frac{125-110 t^{2}+72 t^{3}+9 t^{4}}{\left(t^{2}-5\right)^{2}},
\end{aligned}
$$

$a, t$ - вспомогательные параметры решения. 
Гиперэллиптическая кривая $\widetilde{\Gamma}_{2}^{5}$ в канонической форме имеет следующий вид:

$$
w^{2}=\left(\lambda-\lambda_{1}\right)\left(\lambda-\lambda_{2}\right)\left(\lambda-\lambda_{3}\right)\left(\lambda^{3}+A_{1} \lambda^{2}+B_{1} \lambda+C_{1}\right),
$$

где

$$
\begin{gathered}
\lambda_{1}=6 a \frac{t^{2}+5}{t^{2}-5}, \quad \lambda_{2}=3 a \frac{t^{2}-10 t-15}{t^{2}-5}, \quad \lambda_{3}=3 a \frac{t^{2}+10 t-15}{t^{2}-5}, \\
A_{1}=6 a \frac{11 t^{2}-15}{t^{2}-5}, \quad B_{1}=192 a^{2} \frac{\left(2 t^{2}-5\right)\left(3 t^{2}+5\right)}{\left(t^{2}-5\right)^{2}} \\
C_{1}=144 a^{3} \frac{\left(2 t^{2}-5\right)\left(3 t^{2}+5\right)\left(7 t^{2}-55\right)}{\left(t^{2}-5\right)^{3}}
\end{gathered}
$$

Связь между дополнительньми $(k, \alpha)$ и основными $(\lambda, w)$ спектральными параметрами осушествляется с помошью соотношений

$$
\begin{gathered}
\wp^{\prime}(\alpha)=\frac{16 i w(\lambda-c)\left(\lambda^{2}+A_{3} \lambda+B_{3}\right)\left(\lambda^{2}+A_{4} \lambda+B_{4}\right)}{125\left(\lambda-\lambda_{1}\right)^{2}(\lambda-b)^{3}}, \\
\wp(\alpha)=-\frac{4\left(\lambda^{5}+A_{2} \lambda^{4}+B_{2} \lambda^{3}+C_{2} \lambda^{2}+D_{2}\right)}{25\left(\lambda-\lambda_{1}\right)(\lambda-b)^{2}}, \\
k=\frac{3 i w}{5\left(\lambda-\lambda_{1}\right)(\lambda-b)}, \quad \lambda=\frac{\left(5-t^{2}\right) \partial_{k} \widetilde{\mathscr{R}}(k, \alpha)}{5 a\left\{\left(9 t^{2}-25\right) k^{2}-9 t^{2}\left(\wp(\alpha)-e_{j}\right)\right\}} .
\end{gathered}
$$

Здесь

$$
\begin{gathered}
b=-12 a \frac{t^{2}+5}{t^{2}-5}, \quad c=3 a \frac{7 t^{2}-55}{2\left(t^{2}-5\right)}, \\
A_{2}=15 a \frac{3 t^{2}+5}{t^{2}-5}, \quad B_{2}=5 a^{2} \frac{351 t^{4}+2570 t^{2}-4025}{4\left(t^{2}-5\right)^{2}}, \\
C_{2}=-45 a^{3} \frac{33 t^{6}+1765 t^{4}-3725 t^{2}+22375}{2\left(t^{2}-5\right)^{3}}, \\
D_{2}=\frac{108 a^{5}}{\left(t^{2}-5\right)^{5}} \\
\times\left\{1674 t^{10}-163175 t^{8}+1664000 t^{6}-716250 t^{4}-81250 t^{2}+4665625\right\}, \\
A_{3}=3 a \frac{8 t^{2}-5 t+5}{t^{2}-5}, \quad B_{3}=18 a^{2} \frac{\left(t^{2}-10 t-15\right)\left(8 t^{2}-5 t+5\right)}{\left(t^{2}-5\right)^{2}}, \\
A_{4}=3 a \frac{8 t^{2}+5 t+5}{t^{2}-5}, \quad B_{4}=18 a^{2} \frac{\left(t^{2}+10 t-15\right)\left(8 t^{2}+5 t+5\right)}{\left(t^{2}-5\right)^{2}} .
\end{gathered}
$$

Накрытие (5.1) связано с редукцией абелева голоморфного дифференциала

$$
\frac{5 i \lambda d \lambda}{2 w}=d \alpha
$$

и абелева дифференциала второго рода с простыми полюсами в точках $\mathscr{P}_{\infty}^{ \pm}$

$$
d \Omega_{1}=i\left\{\lambda^{3}+A_{5} \lambda^{2}+B_{5} \lambda+C_{5}\right\} \frac{d \lambda}{w}=-\wp(\alpha) d \alpha+d k,
$$


где

$$
\begin{aligned}
& A_{5}=3 a \frac{9 t^{2}-5}{t^{2}-5} \\
& B_{5}=a^{2} \frac{27 t^{4}+410 t^{2}-6725}{2\left(t^{2}-5\right)^{2}} \\
& C_{5}=-108 a^{3} \frac{\left(t^{2}+15\right)\left(2 t^{2}-5\right)\left(3 t^{2}+5\right)}{\left(t^{2}-5\right)^{3}}
\end{aligned}
$$

\section{6. Новый класс эллиптических потенциалов оператора Дирака}

Примеры, приведенные вьше, показывают, что реально сушествуют кривые Кричевера, принадлежащие анзацу I, и что существует новьй класс эллиптических потенциалов оператора Дирака, не сводящийся к (0.2), (0.3).

Сравнивая анзацы I и II для эллиптических по $x$ решений уравнения НШ $(\mathrm{MKд \Phi ),} \mathrm{легко} \mathrm{заметить} \mathrm{основное} \mathrm{различие} \mathrm{между} \mathrm{соответствующими} \mathrm{накры-}$ вающими отображениями.

Если гиперэллиптическая кривая, ассоциированная с эллиптическим по $x$ решением уравнения НШ (МКд $\Phi)$, удовлетворяет условиям анзаца II, то $\mathfrak{s}\left(\mathscr{P}_{\infty}^{+}\right) \neq$ $\mathfrak{s}(\mathscr{P}-\bar{\infty})$. Поэтому на $\Gamma_{0}$ мы и можем выбрать координату $\alpha$ так, чтобы

$$
\mathfrak{s}\left(\mathscr{P}_{\infty}^{+}\right)=\alpha_{0}, \quad \mathfrak{s}\left(\mathscr{P}_{\infty}^{-}\right)=-\alpha_{0}, \quad \alpha_{0} \neq 0
$$

При этом выполняется, с точностью до голоморфных дифференциалов, равенство (3.13).

$\Phi$ ункции $k(\alpha)$ и $\lambda(\mathscr{P})$ имеют следуюшую асимптотику в окрестности точек $\mathscr{P}_{\infty}^{ \pm}$ (см. (3.9) и (3.11))

$$
\begin{aligned}
& k=\frac{n-1}{\alpha \mp \alpha_{0}} \pm k_{0}+O\left(\alpha \mp \alpha_{0}\right), \quad \mathscr{P} \rightarrow \mathscr{P}_{\infty}^{ \pm}, \\
& \lambda=\mp \frac{i n}{2\left(\alpha \mp \alpha_{0}\right)}+\lambda_{0}+O\left(\alpha \mp \alpha_{0}\right), \quad \mathscr{P} \rightarrow \mathscr{P}_{\infty}^{ \pm} .
\end{aligned}
$$

Если же гиперэллиптическая кривая, ассоциированная с эллиптическим по $x$ решением уравнения НШ (МКд $\Phi)$, удовлетворяет условиям анзаца I, то $\mathfrak{s}\left(\mathscr{P}_{\infty}^{+}\right)=$ $\mathfrak{s}\left(\mathscr{P}_{\infty}^{-}\right)$. В этом случае мы выбираем координату $\alpha \in \Gamma_{0}$ так, чтобы

$$
\mathfrak{s}\left(\mathscr{P}_{\infty}^{+}\right)=\mathfrak{s}\left(\mathscr{P}_{\infty}^{-}\right)=0
$$

Для кривых Кричевера, принадлежащих анзацу I, асимптотика функций $k(\alpha)$ и $\lambda(\mathscr{P})$ будет иметь вид (см. (3.2) и (3.5))

$$
\begin{aligned}
k=\frac{n-2}{2 \alpha} \pm k_{0}+O(\alpha), & \mathscr{P} \rightarrow \mathscr{P}_{\infty}^{ \pm}, \\
\lambda=\mp \frac{i n}{2 \alpha}+\lambda_{0}+O(\alpha), & \mathscr{P} \rightarrow \mathscr{P}_{\infty}^{ \pm} .
\end{aligned}
$$


Одному и тому же накрытию $Г$ (анзац I или II) соответствует бесконечно много бирационально-эквивалентных канонических представлений (1.2) этой гиперэллиптической кривой, отличаюшихся при заффиксированной асимптотике (6.6) лишь сдвигом спектра на произвольную постоянную $\nu \in \mathbb{C}:$

$$
\begin{gathered}
\widetilde{\Gamma}: \widetilde{w}^{2}=\prod_{j=1}^{2 g+2}\left(\widetilde{\lambda}-\widetilde{\lambda}_{j}\right), \\
\widetilde{w}=w, \quad \widetilde{\lambda}=\lambda+\nu, \quad \tilde{\lambda}_{j}=\lambda_{j}+\nu, \quad \widetilde{\lambda}_{0}=\lambda_{0}+\nu .
\end{gathered}
$$

Легко видеть, что векторы $b$-периодов нормированных абелевых дифференциалов второго рода (1.3), определенных на поверхностях $\widetilde{\Gamma}(6.7)$ и Г $(1.2)$, связаны соотношениями:

$$
\widetilde{U}=U, \quad \widetilde{V}=V-4 \nu U, \quad \widetilde{W}=W-6 \nu V+12 \nu^{2} U,
$$

из которых нетрудно вывести закон преобразования потенциала оператора Дирака (анзац I или II) при сдвиге спектра

$$
\begin{aligned}
\widetilde{u}(x, t, y) & =u\left(x-4 \nu t+12 \nu^{2} y, t-6 \nu y, y\right), \\
\widetilde{p}(x, t, y) & =p\left(x-4 \nu t+12 \nu^{2} y, t-6 \nu y, y\right) \exp \left(2 i \nu x-4 i \nu^{2} t+8 i \nu^{3} y\right), \\
\widetilde{q}(x, t, y) & =q\left(x-4 \nu t+12 \nu^{2} y, t-6 \nu y, y\right) \exp \left(-2 i \nu x+4 i \nu^{2} t-8 i \nu^{3} y\right), \quad(6.9) \\
\widetilde{\psi}_{1}(\widetilde{\mathscr{P}}, x, t, y) & =\psi_{1}\left(\mathscr{P}, x-4 \nu t+12 \nu^{2} y, t-6 \nu y, y\right) \exp \left(i \nu x-2 i \nu^{2} t+4 i \nu^{3} y\right), \\
\widetilde{\psi}_{2}(\widetilde{\mathscr{P}}, x, t, y) & =\psi_{2}\left(\mathscr{P}, x-4 \nu t+12 \nu^{2} y, t-6 \nu y, y\right) \exp \left(-i \nu x+2 i \nu^{2} t-4 i \nu^{3} y\right) .
\end{aligned}
$$

Учитьвая все выше сказанное, можно доказать следующее утверждение.

ТЕОРема 4. По любой кривой Кричевера, удовлетворяющей условиям анзача I, мохмно построчть потенциал оператора Дирака, являющийся әллиптической однозначной мероморфной функцией от переменной $x$.

ДокАЗАТЕльство. Если кривая Г является кривой Кричевера, удовлетворяющей условиям анзаца I, то мы можем выбрать соответствующие абелевы дифференциалы так, чтобы они удовлетворяли равенствам (6.4), (3.7). При таком выборе вьполняются все требования теоремы 1 и следствий 1 и 2.

Из следствия 1 вытекает, что (см. (1.5), (2.11), (6.4))

$$
\left\langle S+B_{0} P, D\right\rangle=M_{1}+M_{2} B_{0} .
$$

Таким образом, если из всех бирационально-эквивалентных представлений (1.2) кривой $Г$ взять такое, что постоянное слагаемое $K_{1}$ в асимптотике абелева дифференциала $d \Omega_{1}(1.3)$, определенного на этой кривой, будет равняется

$$
K_{1}=\frac{\pi}{2 \omega}\left(M_{2}-\langle P, D\rangle\right),
$$

то функции $p(x, t, y)$ и $q(x, t, y)$, построенные по этой кривой, будут эллиптическими мероморфными функциями от переменной $x$ :

$$
\begin{array}{ll}
p(x+2 \omega, t, y)=p(x, t, y), & p\left(x+2 \omega^{\prime}, t, y\right)=p(x, t, y), \\
q(x+2 \omega, t, y)=q(x, t, y), & q\left(x+2 \omega^{\prime}, t, y\right)=q(x, t, y),
\end{array}
$$

имеюшими по $n$ нулей и полюсов, а точки ветвления $\lambda_{j}(1.2)$ будут краями зон спектра оператора Дирака с данным потенциалом.

Равенства (6.12) являются следствием соотношений (1.5), (2.13), (6.9) и (6.11). 
ЗАмЕчАниЕ 7. Если конечнозонный потенциал оператора Дирака будет строиться по кривой Кричевера, удовлетворяющей условиям анзаца II, то функции $p(x, t, y)$ и $q(x, t, y)$ будут эллиптическими функциями второго рода от переменной $x$ :

$$
\begin{aligned}
& p(x+2 \omega)=p(x), \quad p\left(x+2 \omega^{\prime}\right)=p(x) \exp \left\{-\frac{2 \pi i \alpha_{0}}{\omega}\right\}, \\
& q(x+2 \omega)=q(x), \quad q\left(x+2 \omega^{\prime}\right)=q(x) \exp \left\{\frac{2 \pi i \alpha_{0}}{\omega}\right\} .
\end{aligned}
$$

Законы преобразования потенциала при сдвиге аргумента $x$ на периоды тора приведены для спектральной кривой, удовлетворяюшей условию (6.11).

СлЕДСтвИЕ 4 . В условиях теоремъ 2 әллиптический потенциал оператора Дирака имеет вид:

$$
\begin{gathered}
p(x, t, y)=p_{0}(t, y)+\sum_{j=1}^{n} \mu_{j}^{-1}(t, y) \zeta\left(x-x_{j}(t, y)\right), \\
q(x, t, y)=q_{0}(t, y)+\sum_{j=1}^{n} \mu_{j}(t, y) \zeta\left(x-x_{j}(t, y)\right), \\
\sum_{j=1}^{n} \mu_{j}(t, y)=0, \quad \sum_{j=1}^{n} \mu_{j}^{-1}(t, y)=0,
\end{gathered}
$$

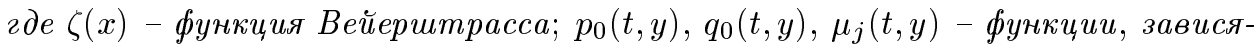
щие от параметров $t, y ; x_{j}(t, y)$ - полюсы потенциала.

ДокАЗАТЕЛЬство следует из соотношений (1.5), равенства (1.7), которое в случае эллиптического потенциала (анзац I или II) примет вид (3.4), и свойств эллиптических функций.

СлЕДСтвиЕ 5. Определим вспомогательнье функиии $p_{0 j}(t, y)$ и $q_{0 j}(t, y)$ :

$$
\begin{aligned}
& p_{0}(t, y)=\mu_{j}^{-1} p_{0 j}(t, y)+\sum_{k \neq j} \mu_{k}^{-1} \zeta\left(x_{k}(t, y)-x_{j}(t, y)\right), \\
& q_{0}(t, y)=\mu_{j} q_{0 j}(t, y)+\sum_{k \neq j} \mu_{k} \zeta\left(x_{k}(t, y)-x_{j}(t, y)\right) .
\end{aligned}
$$

Тогда полюсы $x_{j}(t, y)$ әллиптического потенциала (6.14) оператора Дирака для любого $j=1, \ldots, n$ и для любых значений параметров $t$, у удовлетворяют уравнениям

$$
\begin{gathered}
\sum_{k \neq j}\left(\frac{\mu_{k}}{\mu_{j}}+1+\frac{\mu_{j}}{\mu_{k}}\right) \wp\left(x_{k}(t, y)-x_{j}(t, y)\right)=\varkappa+p_{0 j} q_{0 j} \\
p_{0 j}+q_{0 j}=0 .
\end{gathered}
$$

ДокАЗАТЕЛЬСтво. Подставим (6.14) в (3.4). Уравнения (6.16), (6.17) получаются приравниванием соответствующих коэффициентов в асимптотиках левой и правой частей равенства (3.4) в окрестности полюсов $x=x_{j}(t, y), j=1, \ldots, n$. 
ЗАмечАниЕ 8. Уравнения (6.14), (6.16), (6.17) являются необходимыми, но не достаточными условиями сушествования конечнозонного эллиптического потенциала оператора Дирака.

ТеОрема 5. Полюсы $x_{j}(t)$ конечнозонного әллиптического решения уравнения НШІ (анзаи I) удовлетворяют динамике интегрируемой системь частии, Калоджеро-Мозера (0.5), а функиии $p_{0 j}(t)$ (6.15) являются, с точностью до постоянного множителя, импульсами частии:

$$
\dot{x}_{j}(t)=-2 i p_{0 j}(t)
$$

ДоКАЗАТЕЛЬСТво нетрудно получить, подставив анзац (6.14), (6.15) в уравнение (1.1) и использовав свойства эллиптических функций.

ЗАмЕчАнИЕ 9. Эволюция полюсов двухзонных решений уравнения НШ, построенных по кривым (4.2) или (5.2), описывает динамику некоторых систем Калоджеро-Мозера из пяти частиц.

\section{7. Примеры эллиптических мероморфных потенциалов оператора Дирака}

Проше всего построить 2-эллиптические потенциалы, т.е. эллиптические потенциалы с двумя полюсами, так как двулистные накрытия анзаца I должны удовлетворять меньшим требованиям, чем накрытия с большим числом листов, поскольку при $n=2$ уравнение (3.7) переходит в (3.7а).

Очевидно, что для $n=2$ род поверхности не может быть больше $g=2$, поскольку для анзаца I число неподвижных относительно гиперэллиптической инволюции $\tau:(k, \alpha) \rightarrow(-k,-\alpha)$ точек (т.е. точек ветвления гиперэллиптической кривой) не может превосходить $N=6$.

ПримеР 1. Нетрудно показать, что однозонный 2-эллиптический потенциал имеет вид (с точностью до сдвига по переменной $x$ ):

$$
\begin{aligned}
& u(x, 0)=\wp(x)+\wp\left(x-\omega_{j}\right)+e_{j}, \\
& p(x, 0)=\mu^{-1}\left\{\zeta(x)-\zeta\left(x-\omega_{j}\right)-\eta_{j}\right\}, \\
& q(x, 0)=\mu\left\{\zeta(x)-\zeta\left(x-\omega_{j}\right)-\eta_{j}\right\} .
\end{aligned}
$$

Здесь $\eta_{j}=\zeta\left(\omega_{j}\right), e_{j}=\wp\left(\omega_{j}\right) ; \zeta(x), \wp(x)$ - эллиптические функции Вейерштрасса с периодами накрьваемого тора.

Потенциалу (7.1) соответствует спектральная кривая

$$
\Gamma: w^{2}=\lambda^{4}-3 e_{j} \lambda^{2}+H_{j}^{2},
$$

где $H_{j}^{2}=\left(e_{j}-e_{k}\right)\left(e_{j}-e_{l}\right)=3 e_{j}^{2}-g_{2} / 4$.

Также нетрудно найти динамику потенциала (7.1) в силу уравнения НШ (1.1)

$$
\begin{gathered}
p(x, t)=p(x, 0) \exp \left\{-6 i e_{j} t\right\}, \quad q(x, t)=q(x, 0) \exp \left\{6 i e_{j} t\right\}, \\
u(x, t)=u(x, 0) .
\end{gathered}
$$


ПримеР 2. Двузонньй 2-эллиптический потенциал оператора Дирака (анзацI) имеет вид:

$$
\begin{aligned}
& u(x, 0)=\wp\left(x-x_{1}\right)+\wp\left(x-x_{2}\right)-\frac{1}{3} \gamma_{2}, \\
& p(x, 0)=\mu^{-1}\left\{\zeta\left(x-x_{1}\right)-\zeta\left(x-x_{2}\right)+\zeta\left(x_{1}-x_{2}\right)+\rho\right\} \\
& q(x, 0)=\mu\left\{\zeta\left(x-x_{1}\right)-\zeta\left(x-x_{2}\right)+\zeta\left(x_{1}-x_{2}\right)-\rho\right\} .
\end{aligned}
$$

Здесь

$$
\gamma_{2} \neq-3 e_{j}, \quad \wp\left(x_{1}-x_{2}\right)=\rho^{2}-\frac{1}{3} \gamma_{2} .
$$

Детально исследуя этот потенциал, можно получить следующие ограничения на спектральную кривую $\Gamma(1.2)$ :

$$
\begin{aligned}
\gamma_{1} & =0, \quad \gamma_{3}=0, \quad \gamma_{5}=0 \\
g_{2} & =\frac{4}{3} \gamma_{2}^{2}-4 \gamma_{4} \\
g_{3} & =\frac{8}{27} \gamma_{2}^{3}-\frac{4}{3} \gamma_{2} \gamma_{4}+4 \gamma_{6} .
\end{aligned}
$$

Из (7.6) следует, что кривая Г задается уравнением:

$$
\Gamma: w^{2}=\lambda^{6}+\gamma_{2} \lambda^{4}+\gamma_{4} \lambda^{2}+\gamma_{6},
$$

анализируя которое, нетрудно найти конкретный вид накрываюшего отображения $\mathfrak{s}:(w, \lambda) \rightarrow \alpha$,

$$
\wp^{\prime}(\alpha)=2 i w, \quad \wp(\alpha)=-\lambda^{2}-\frac{1}{3} \gamma_{2}, \quad d \alpha=i \frac{\lambda d \lambda}{w} .
$$

При $\gamma_{2}=-3 e_{j}$ накрывающая кривая вырождается до эллиптической $(7.2)$, а накрываюшее отображение $\mathfrak{s}:(w, \lambda) \rightarrow \alpha$ описывается равенствами

$$
\wp^{\prime}(\alpha)=2 i \lambda w, \quad \wp(\alpha)=-\lambda^{2}+e_{j}, \quad d \alpha=i \frac{d \lambda}{w} .
$$

Известно (см., например, [32], [7], [10], [18]), что если кривая Г рода $g=2$ является накрытием над эллиптической кривой $\Gamma_{0}$, то

а) она также является накрытием и над другой эллиптической кривой $\widetilde{\Gamma}_{0}$;

б) оба накрытия имеют одинаковое число листов.

Как и в случае уравнения Кд $\Phi$, динамика двухзонных эллиптических решений уравнения НШ может быть найдена только с помощью второго накрываемого тоpa $\widetilde{\Gamma}_{0}$.

Кривая (7.7) является хорошо изученной [36], [37], [7], [10]. Для нее второе накрывающее отображение $\mathfrak{s}_{1}: \Gamma \rightarrow \widetilde{\Gamma}_{0}$,

$$
\widetilde{\Gamma}_{0}: \chi^{2}=\left(E+\frac{1}{3} \gamma_{2}\right)\left(4 E^{3}-g_{2} E-g_{3}\right),
$$


имеет вид:

$$
\chi=-2 \lambda w, \quad E=-\lambda^{2}-\frac{1}{3} \gamma_{2}, \quad \frac{d E}{\chi}=\frac{d \lambda}{w} .
$$

Подставляя анзац (7.3) в уравнение (1.1), получаем временну́ю зависимость полюсов эллиптического потенциала (7.3) оператора Дирака в силу уравнения НШ:

$$
\dot{x}_{1}=-2 i \rho, \quad \dot{x}_{2}=2 i \rho, \quad \partial_{t} \ln \mu=i\left(4 \wp\left(x_{1}-x_{2}\right)-\frac{2}{3} \gamma_{2}\right) .
$$

Из равенств (7.12) вытекает, что "центр масс" полюсов неподвижен:

$$
\partial_{t}\left(x_{1}+x_{2}\right)=0
$$

и, следовательно, для описания динамики достаточно найти временну́ю зависимость разности полюсов $x_{21}=x_{2}-x_{1}$.

Пусть $(\chi(\mathscr{E}), \mathscr{E})$ - точка на торе $\widetilde{\Gamma}_{0}$ такая, что

$$
\mathscr{E}=\wp\left(x_{21}\right) \text {. }
$$

Из (7.5) и (7.12) следует, что $\mathscr{E}(t)$ удовлетворяет дифференциальному уравнению

$$
\dot{\mathscr{E}}=4 i \chi(\mathscr{E})
$$

которое имеет решение

$$
\int_{\mathscr{E}(0)}^{\mathscr{E}(t)} \frac{d E}{\chi}=4 i t
$$

Зависимость $\mathscr{E}(t)$ можно получить, обратив эллиптический интеграл, стоящий в левой части уравнения (7.14).

Из соотношений (7.12)-(7.14) вытекает динамика полюсов $x_{j}(t)$ :

$$
x_{1}(t)=x_{1}(0)-\frac{1}{2} \int_{\mathscr{E}(0)}^{\mathscr{E}(t)} \frac{d \wp}{\wp^{\prime}}, \quad x_{2}(t)=x_{2}(0)+\frac{1}{2} \int_{\mathscr{E}(0)}^{\mathscr{E}(t)} \frac{d \wp}{\wp^{\prime}}
$$

и зависимость $\mu(t)$ :

$$
\mu(t)=\mu_{0} \exp \left\{i \int_{\mathscr{E}(0)}^{\mathscr{E}(t)}\left(4 E-\frac{2}{3} \gamma_{2}\right) \frac{d E}{\chi}\right\}
$$

которые дают полное описание двузонного эллиптического решения уравнения НШ с начальньми данньми (7.4).

Из теоремы 5 вытекает, что соотношения (7.14), (7.15) описывают динамику на торе $\Gamma_{0}$ системы Калоджеро-Мозера из двух частиц [38]. Края зон эллиптической кривой $\widetilde{\Gamma}_{0}$, ответственной за динамику, определяются параметрами тора $\Gamma_{0}$, начальными значениями $x_{1}(0), x_{2}(0)$ и начальными импульсами $\dot{x}_{1}(0)=-\dot{x}_{2}(0)$ и легко могут быть получены с помощью соотношений (7.5), (7.6), (7.12).

Для того чтобы определить динамику системы Калоджеро-Мозера из двух частиц с начальными импульсами $\dot{x}_{1}(0) \neq-\dot{x}_{2}(0)$, надо сдвинуть края зон (см. равенства (6.9)) накрывающей кривой $Г(7.7)$ на $\nu$ :

$$
\nu=\frac{\dot{x}_{1}(0)+\dot{x}_{2}(0)}{8} .
$$

В этом случае к скорости каждого из полюсов добавится постоянная составляющая равная $4 \nu$. 


\section{Список литературы}

1. Дубровин Б. А., Новиков С. П. Периодический и условно периодический аналоги многосолитонных решений уравнения Кортевега-де Фриза // ЖЭЭТФ. 1974. Т. 67. №12. C. 2131-2143.

2. Airault H., McKean H.P., Moser J. Rational and elliptic solutions of the KdV equation // Comm. Pure Appl. Math. 1977. V. 30. P. 95-148.

3. Кричевер И. М. Эллиптические решения уравнения Кадомцева-Петвиашвили и интегрируемые системы частиц // Функцион. анализ и его прил. 1980. Т. 14. № 4. С. 45-54.

4. Итс А. Р., Энольский В. З. О динамике системы Калоджеро-Мозера и редукции гиперэллиптических интегралов к эллиптическим интегралам // Функцион. анализ и его прил. 1986. Т. 20. №1. С. $73-74$.

5. Eilbeck J.C., Enol'skii V.Z. Elliptic Baker-Akhiezer functions and application to an integrable dynamical system // J. Math. Phys. 1994. V. 35. № 3. P. 1192-1201.

6. Переломов A.M. Интегрируемые системы классической механики и алгебры Ли. М.: Наука, 1990.

7. Белоколос Е.Д., Бобенко А.И., Матвеев В. Б., Энольский В.З. Алгеброгеометрические принципы суперпозиции конечнозонных решений интегрируемых нелинейных уравнений // УМН. 1986. Т. 41. № 2. С. 3-42.

8. Белоколос Е. Д., Энольский В.З. Изоспектральные деформации эллиптических потенциалов // УМН. 1989. Т. 44. №5. С. 155-156.

9. Белоколос Е. Д., Энольский В. З. Эллиптические солитоны Верде и теория редукции Вейерштрасса // Функцион. анализ и его прил. 1989. Т. 23. № 1. С. 57-58.

10. Belokolos E.D., Bobenko A.I., Enol'skii V.Z., Its A.R., Matveev V.B. Algebrogeometrical approach to nonlinear evolution equations // Springer Ser. Nonlinear Dynam. 1994.

11. Смирнов А. О. Оператор Дирака с эллиптическим потенциалом // Матем. сб. 1995. T. 186. № 8. C. 134-141.

12. Gerdt V.P., Kostov N.A. Computer algebra in the theory of ordinary differential equations of Halphen type // Computers and Mathematics / ed. E. Kaltofen, S.M. Watt. Berlin: Springer-Verlag, 1989. P. 277-283.

13. Enol'skii V.Z., Kostov N.A. On the geometry of elliptic solitons // Acta Appl. Math. 1994. V. 36. P. 57-86.

14. Brezhnev Yu. V. Darboux transformation and some multi-phase solutions of the DoddBullough-Tzitzeica equation: $U_{x t}=e^{U}-e^{-2 U} / /$ Phys. Lett. A. 1996. V. 211. P. 94-100.

15. Смирнов А.О. Эллиптические решения уравнения Кортевега-де Фриза // Матем. заметки. 1989. Т. 45. №6. С. 66-73.

16. Смирнов А. О. Эллиптические решения интегрируемых нелинейных уравнений // Матем. заметки. 1989. Т. 46. № 5. С. 100-102.

17. Смирнов А. О. Эллиптические по $t$ решения уравнения КдФ // ТМФ. 1994. Т. 100. № 2 . C. $183-198$.

18. Smirnov A. O. Finite-gap elliptic solutions of the KdV equation // Acta Appl. Math. 1994. V. 36. P. $125-166$.

19. Смирнов A. O. Двухзонные эллиптические решения интегрируемых нелинейных уравнений // Матем. заметки. 1995. Т. 58. №1. С. 86-97.

20. Смирнов А.О. Вещественные эллиптические решения уравнения "sine-Gordon" // Матем. сб. 1990. Т. 181. №6. С. 804-812.

21. Смирнов А. О. Эллиптические решения нелинейного уравнения Шрёдингера и модифицированного уравнения Кортевега-де Фриза // Матем. сб. 1994. Т. 185. № 8. С. 103-114.

22. Смирнов $A$. О. Эллиптические по $t$ решения нелинейного уравнения Шрёдингера // ТMФ. 1996. T. 107. № 2. C. 188-200.

23. Gesztesy F., Weikard R. On Picard potentials // Preprint, 1994.

24. Gesztesy F., Weikard R. Picard potentials and Hill's equation on torus // Preprint, 1994.

25. Gesztesy F., Weikard R. Lamé potentials and the stationary (m)KdV hierarchy // Preprint, 1994. 
26. Gesztesy F., Weikard R. Treibich-Verdier potentials and the stationary (m)KdV hierarchy // Preprint, 1994.

27. Ахиезер Н. И. Элементы теории эллиптических функций. М.: Наука, 1970.

28. Итс A.P. Обращение гиперэллиптических интегралов и интегрирование нелинейных дифференциальных уравнений // Вестник ЛГУ. Сер. Матем.-мех.-астр. 1976. Т. 7. № 2 . C. $39-46$.

29. Итс A. Р., Котляров В. П. Об одном классе решений нелинейного уравнения Шрёдингера // ДАН УССР. Сер. А. 1976. № 11. С. 965-968.

30. Иmс А.P. Точное интегрирование в римановых $\Theta$-функциях нелинейного уравнения Шрёдингера и модифицированного уравнения Кортевега-де Фриза // Дис. ... канд. физ.-матем. наук. Л.: ЛГУ, 1977.

31. Matveev V. B. Abelian functions and solitons // Preprint № 373. Univ. of Wrocław, 1976.

32. Krazer A. Lehrbuch der Thetafunktionen. Leipzig: Teubner, 1903.

33. Зверович Э.И. Краевые задачи теории аналитических функций в гельдеровских классах на римановых поверхностях // УМН. 1971. Т. 26. № 1. С. 113-179.

34. Дубровин Б. А. Тэта-функции и нелинейные уравнения // УМН. 1981. Т. 36. № 2. C. $11-80$.

35. Мамфорд Д. Лекции о тэта-функциях. М.: Мир, 1988.

36. Бабич М.В., Бобенко А.И., Матвеев В. Б. Редукции многомерных тэта-функций и симметрии алгебраических кривых // ДАН СССР. 1983. Т. 272. № 1. С. 13-17.

37. Бабич M. В., Бобенко А. И., Матвеев В. Б. Решения нелинейных уравнений, интегрируемых методом обратной задачи, в тэта-функциях Якоби и симметрии алгебраических кривых // Изв. АН СССР. Сер. матем. 1985. Т. 49. № 3. С. 511-529.

38. Choodnovsky D. V. Meromorphic solutions of nonlinear partial differential equations and particle integrable systems // J. Math. Phys. 1979. V. 20. № 12. P. 2416-2424.

С.-Петербургская государственная академия

аэрокосмического приборостроения

Поступила в редакцию

31.10.1995 